\title{
Retrieved emotional context influences hippocampal involvement during recognition of neutral memories
}

\author{
Atsuko Takashima ${ }^{\mathrm{a}, \mathrm{b}, *}$, Frauke van der Ven ${ }^{\mathrm{a}, \mathrm{b}}$, Marijn C.W. Kroes ${ }^{\mathrm{a}, \mathrm{c}}$, Guillén Fernández ${ }^{\mathrm{a}, \mathrm{d}}$ \\ a Donders Institute for Brain, Cognition and Behaviour, Radboud University, Nijmegen, The Netherlands \\ b Behavioural Science Institute, Radboud University, Nijmegen, The Netherlands \\ ${ }^{c}$ Department of Psychology, and Center for Neural Science, New York University, New York, NY, USA \\ ${ }^{\mathrm{d}}$ Radboud University Medical Centre, Department for Cognitive Neuroscience, Nijmegen, The Netherlands
}

\section{A R T I C L E I N F O}

\section{Article history:}

Received 12 February 2016

Accepted 31 August 2016

Available online 1 September 2016

\section{Keywords:}

Hippocampus

Emotional memory enhancement

Memory consolidation

fMRI

Context memory

\begin{abstract}
A B S T R A C T
It is well documented that emotionally arousing experiences are better remembered than mundane events. This is thought to occur through hippocampus-amygdala crosstalk during encoding, consolidation, and retrieval. Here we investigated whether emotional events (context) also cause a memory benefit for simultaneously encoded non-arousing contents and whether this effect persists after a delay via recruitment of a similar hippocampus-amygdala network. Participants studied neutral pictures (content) encoded together with either an arousing or a neutral sound (that served as context) in two study sessions three days apart. Memory was tested in a functional magnetic resonance scanner directly after the second study session. Pictures recognised with high confidence were more often thought to have been associated with an arousing than with a neutral context, irrespective of the veridical source memory. If the retrieved context was arousing, an area in the hippocampus adjacent to the amygdala exhibited heightened activation and this area increased functional connectivity with the parahippocampal gyrus, an area known to process pictures of scenes. These findings suggest that memories can be shaped by the retrieval act. Memory structures may be recruited to a higher degree when an arousing context is retrieved, and this may give rise to confident judgments of recognition for neutral pictures even after a delay.
\end{abstract}

(c) 2016 Elsevier Inc. All rights reserved.

\section{Introduction}

Memories for emotional or arousing events are remembered well and are often highly vivid even long after their occurrence (for reviews: Cahill and McGaugh (1998), Christianson (1992), Kensinger and Corkin (2004), LaBar and Cabeza (2006), Phelps (2006)). The hippocampus is a critical brain structure for encoding and retrieval of newly learned information (Alvarez and Squire, 1994; Moscovitch et al., 2005; Nadel and Moscovitch, 1997; Squire, 1992). The amygdala is considered to up-regulate hippocampal processing during encoding, consolidation (McGaugh, 2006; Richardson et al., 2004), and retrieval (Dolcos et al., 2005; Ritchey et al., 2008; Sharot et al., 2007; Smith et al., 2005, 2006, 2004; Sterpenich et al., 2009) leading to enhanced memory for arousing

Abbreviations: AAL, automated anatomical labelling; E-, encoding; FWE, familywise error corrected; ITI, inter-trial interval; MNI, Montreal Neurological Institute: PPI, psycho-physiological interaction; R-, retrieval; ANOVA, analysis of variance; ROI, region of interest; SVC, small volume correction.

* Corresponding author at: Donders Institute for Brain, Cognition and Behaviour, Radboud University,PO box 9101, 6500 HB Nijmegen, The Netherlands.

E-mail address: Atsuko.Takashima@donders.ru.nl (A. Takashima). events (LaBar and Phelps, 1998). However, the neural mechanisms via which emotion contributes to enhanced memory retrieval are unclear as they are difficult to isolate. First, emotional retrieval cues can inherently evoke online processing of sensory information that in turn evokes emotional responses and can influence retrieval. Second, mnemonic mechanisms may reinstate an emotional experience that occurred during encoding and influence retrieval. One way to isolate the neural mechanisms underlying the retrieval of emotion from perception evoked responses is to use neutral cues that have been associated with emotional context during encoding to test the emotional memory.

Memories for neutral content can be influenced by an arousing context at encoding. For instance, emotional context, such as emotional background pictures and emotional context sentences, can positively influence the recognition of neutral pictures/words embedded in these emotional contexts when tested in short succession (Maratos and Rugg, 2001; Smith et al., 2004). The previous studies also investigated the neural responses at retrieval shortly after encoding. They observed heightened involvement of the hippocampus and amygdala for retrieval of neutral contents embedded in emotional contexts, which is suggestive of enhanced memory and emotion processing at the time of retrieval for 
neutral items arising from their study history. For a longer delay, memory persistence for neutral items encoded under a threat of shock has been shown (Dunsmoor et al., 2014, 2015), but less is known about the neural responses at the time of delayed retrieval.

Retrieved memories are not always veridical. Memories of emotionally arousing events are often vivid, detailed and accompanied by a high feeling of confidence, despite potential inaccuracy (Neisser and Harsch, 1992; Talarico and Rubin, 2003). Thus, if a retrieved memory is thought to have been encoded in an arousing context, this may enhance the subjective confidence for the retrieved memory, even though this may not result from a proper reconstruction of the past event. The hippocampus has been reported to show more activity when the retrieved memory is accompanied by a feeling of recollection compared to a feeling of familiarity (Diana et al., 2007a, 2007b; Weis et al., 2004). Moreover, item-emotion binding via the amygdala has been suggested to be the key for persistence of emotional memories (Yonelinas and Ritchey, 2015). This raises the question whether hippocampal and amygdala involvement at retrieval may be influenced by the retrieved emotional association independent of veridical accuracy.

In the current study, we asked whether an arousing context at encoding influences the retrieval of memory for associated neutral content, and whether this potential influence changes as a function of time. Furthermore, we asked whether the memory of neutral content is modulated by the encoded and retrieved context. To address these questions, participants studied two sets of neutral pictures (content) experienced together with either an unpleasant arousing or a neutral non-arousing sound (context) at two occasions three days apart. To observe neural correlates of retrieval and the possible changes with time, we tested recognition memory for the pictures (content memory) and cued-recall of the associated sounds (context memory) for both recently (same day) and remotely (three days earlier) studied stimulus sets. Neural responses were recorded using functional magnetic resonance imaging (fMRI) during the retrieval session. We predicted enhanced memory for neutral content encoded within an arousing context over a non-arousing context, and possible influences of the encoded and retrieved context on the neural mechanisms associated with retrieval of neutral content.

\section{Materials and methods}

\subsection{Participants}

Twenty-four healthy, right-handed, male participants $(M=22.1$ years, $S D=2.8$, range: $18-28$ years) with no neurological or psychiatric history were recruited from the university campus in Nijmegen. We restricted our participant population to males to avoid potential gender lateralisation (Cahill, 2010) and possible effects of the menstrual cycle in women (Toffoletto et al., 2014). All participants provided written informed consent and were compensated with either money or study credits. The study was approved by the institutional ethics committee (CMO Region Arnhem-Nijmegen, the Netherlands). Due to too few trials (less than 5 ) in one or more of the critical conditions of interest, data of four participants were excluded from the fMRI analyses.

\subsection{Procedure}

\subsubsection{Day 1: remote memory encoding and sound rating}

Upon arrival, participants were given an overview of the experiment. Next, participants intentionally encoded a set of picturesound associations. The neutral pictures were delivered together with either an unpleasant arousing or a neutral non-arousing sound by a computer in a sound attenuated room. Participants were instructed to remember the picture-sound associations and were explicitly told that their memory would be tested later, on Day 1 and again on Day 4 . The sounds were presented through a headphone and the volume was adjusted individually to a comfortable volume level for each participant. Twenty pictures of landscapes were with- and 20 were without buildings. Each of these pictures were paired with one of the 10 unpleasant arousing sounds (resulting in each sound being associated to four different pictures). Another set of 40 pictures (20 pictures with- and 20 without buildings) were paired with one of 10 the neutral nonarousing sounds. Thus there were a total of 80 picture-sound pairs for the Day 1 encoding session (see Section 2.3 below). Prior to the encoding session, a practice session of six trials was given to acquaint the participant with the task using additional stimuli not used in the actual experiment.

The encoding session consisted of two runs and each pair appeared once in each run (Fig. 1A and B). For every trial, after an inter-trial interval (ITI) of $1 \mathrm{~s}$, a picture appeared on screen for $3 \mathrm{~s}$, of which the last $1 \mathrm{~s}$ overlapped with the sound stimulus, similar to what is done in delay conditioning paradigms (Knight et al., 2004). At the offset of the sound, participants were prompted to provide a response within a time limit of $2 \mathrm{~s}$. During the first run, participants were asked to indicate whether the picture included a building (yes=left control key, no=right control key), and during the second run, they were asked to rate whether the sound was arousing or not (yes=left, no=right). The response to this question during the second run was used to categorise Encoded context as either Arousing or Neutral. To avoid rapid switching from arousing to neutral context, the trials were presented in a pseudorandom order such that three to five trials with the same sound category (Arousing/Neutral) were blocked together. Between blocks, an extra inter-block interval of $4 \mathrm{~s}$ was inserted. There was a break of 5 min between the first and the second run. We opted to have two encoding sessions to reinforce learning of the picturesound pairs as to ensure memory retention after a $72 \mathrm{~h}$ delay. Furthermore, by probing the orientation question to both the picture (building/landscape) and the sound (arousing/neutral), we hoped that participants would pay attention to both the visual and the auditory information of the studied material.

In order to validate whether our choice of sound context was according to the experimental manipulation, participants rated each sound presented during the encoding session again during the sound rating session, but this time on two dimensions in two runs. In the first run, participants rated on 6-point Likert scales the pleasantness of the sounds ( $1=$ pleasant, $6=$ unpleasant), and in the second run the arousal level of the sounds $(1=\mathrm{calm}$, $6=$ arousing). Participants responded at their own pace during this session.

\subsubsection{Day 4: recent memory encoding, sound rating, and retrieval} test

Three days after the remote memory encoding session, participants returned to the laboratory for a recent encoding session following the same routine as on Day 1 . First, subjects studied a second set of 80 novel picture-sound associations (different pictures from the remote set, but the same sounds as the remote set). Next, they rated the sounds again identical to the procedure on Day 1.

After a 15-min break, subjects entered the magnetic resonance imaging (MRI) scanner for a recognition test (Fig. 1C). All pictures from the remote $(N=80$, encoded on Day 1$)$ and recent $(N=80$, encoded on Day 4) sets, and another set of pictures (new, $N=80$ ) were presented once each in random order. Each trial started with a picture appearing on the screen for $2 \mathrm{~s}$. To assess content memory, participants were prompted to indicate via a button press whether they thought the picture was old (studied in one of the 


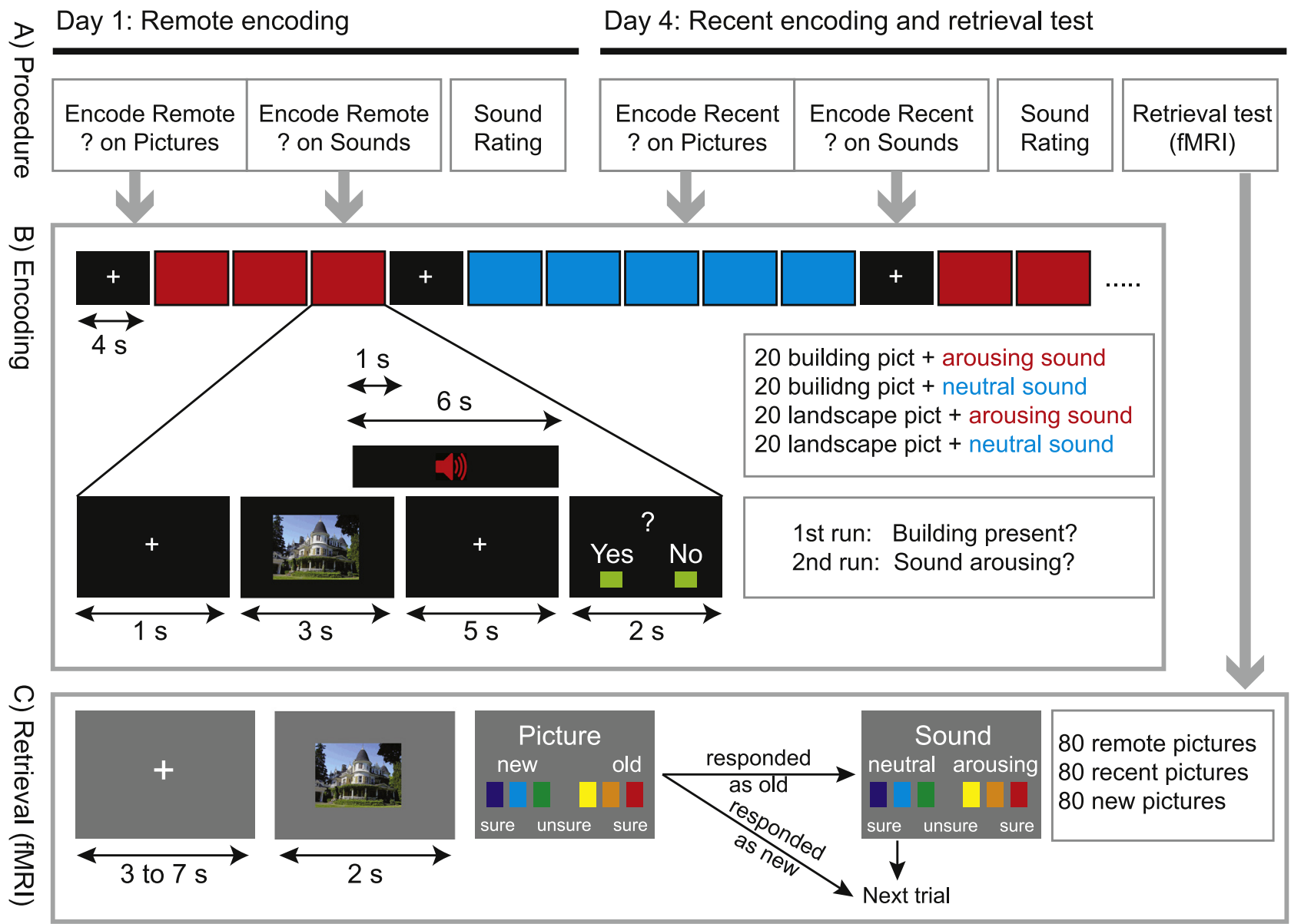

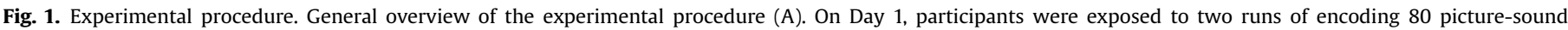

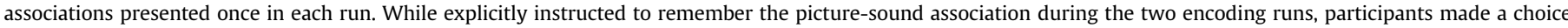

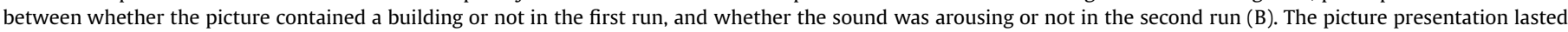

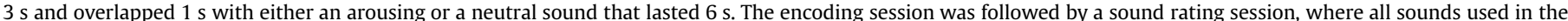

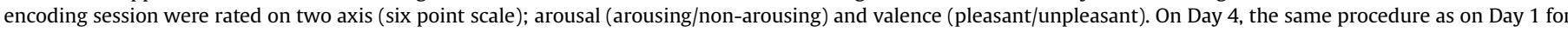

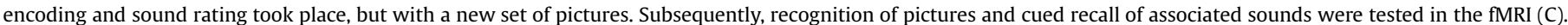

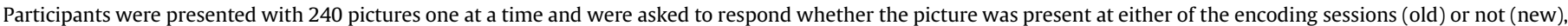

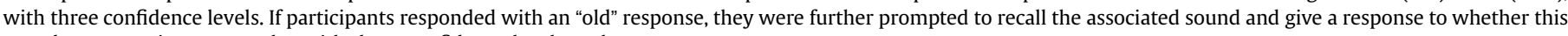
sound was arousing or not, also with three confidence levels each.

encoding sessions) or new (not studied before), each with three confidence levels (very sure, sure, unsure), providing six response options in total. In case participants indicated the picture to be old (independent of the confidence level), the context memory was probed with a second question asking whether they thought the picture was paired with an arousing or a neutral sound at encoding, also on a three-level confidence scale. Each trial was separated by a jittered ITI of 3 to $7 \mathrm{~s}$ during which a white fixation cross on a grey background was shown on the screen. Twenty 'null events' consisting of the same white fixation cross as the ITI presented for $10 \mathrm{~s}$ were randomly interspersed between the trials, to serve as a baseline condition. The retrieval session started with two filler trials, followed by the experimental trials.

Following the retrieval task, subjects performed a localiser task inside the MRI scanner. Participants were presented with a sequential series of pictures presented in blocks. The pictures consisted of 60 landscapes, 60 faces, and 60 scrambled pictures, not included in the memory task. Each block consisted of 10 pictures from one category. Every picture was presented for $1 \mathrm{~s}$ with an ITI of $500 \mathrm{~ms}$. Within a block, two of the ten pictures repeated on a subsequent trial and the participants were instructed to press a button whenever a presented picture was identical to the one before. A $10 \mathrm{~s}$ interval during which a fixation cross was shown in the center of the screen separated each block. Lastly, a structural scan was acquired.

\subsection{Stimuli}

\subsubsection{Visual stimuli}

A pool of 368 photos of landscapes was rated by four independent raters on arousal, distinctiveness, and expected memorability. The 240 most neutral pictures were chosen for the stimulus set, and their luminance was adjusted to have a similar overall luminance across images using in-house software (Matlab 2009b; MathWorks). Pictures were divided into three sets of 80 ( 40 with buildings and 40 without buildings in each set), which were matched on stimulus complexity and balanced for types of content, and assigned to the remote, recent, or new condition. The assignment of the sets to the conditions was counterbalanced across subjects. An additional 14 neutral landscape photographs were used either during practice sessions or as filler trials in the scanner, but were not considered in the analyses.

\subsubsection{Auditory stimuli}

Twenty sounds were selected from the International Affective Digitized Sounds (IADS-2; Bradley and Lang, 2007). Based on the IADS ratings, 10 unpleasant arousing sounds (Arousing), and 10 
neutral non-arousing sounds (Neutral) were selected (on a 9 point Likert scale with low values indicating non-arousing/unpleasantness: $M \pm S D$ Arousing: arousal (rated by males) $7.33 \pm 0.47$, valence $2.55 \pm 0.52$, Neutral: arousal $4.04 \pm 0.52$, valence $5.27 \pm 0.56$ ). The stimuli included animal, human, natural, and artificial sounds and the number of sound stimuli in these categories was matched in the Neutral and Arousing set. All 20 sound stimuli were six seconds in length. Two arousing sounds and five neutral sounds, not included in the above sets, were used for the practice sessions. Sound volume was normalised using Normalize 0.7.7 (http://normalize.nongnu.org).

\subsection{MRI acquisition and data analyses}

MRI data were acquired with a $1.5 \mathrm{~T}$ Siemens Avanto MR scanner (Siemens, Erlangen, Germany), equipped with an 8 channel head coil. For functional scans, T2*-weighted images covering the whole brain were acquired using an echo-planar imaging sequence (EPI, 31 axial slices, ascending slice acquisition, repetition time $(T R)=2.28 \mathrm{~s}$, echo time $(\mathrm{TE})=35 \mathrm{~ms}, 90^{\circ}$ flip-angle, slice matrix $=64 \times 64$, slice thickness: $3.5 \mathrm{~mm}$, slice gap: $0.35 \mathrm{~mm}$, field of view: $212 \times 212 \mathrm{~mm}$. For the structural scan, T1-weigted images were acquired by using magnetisation-prepared, rapid acquisition gradient echo sequence (MP-RAGE, 176 sagittal slices, $\mathrm{TR}=2250 \mathrm{~ms}, \mathrm{TE}=2.95 \mathrm{~ms}, 15^{\circ}$ flip-angle, matrix $=256 \times 256$, slice thickness: $1.0 \mathrm{~mm}$, FOV: $256 \times 256 \mathrm{~mm}$ ).

Image pre-processing and analyses were performed using SPM8 (www.fil.ion.ucl.ac.uk). The first five functional volumes were discarded to allow for $\mathrm{T} 1$ equilibration, and the remaining images were realigned to the mean volume. The subject-mean functional image was co-registered with the corresponding structural MRI. After the images were slice time corrected to the 16th slice, both functional and structural scans were spatially normalised to the Montreal Neurological Institute (MNI) T1 template, resampled into $2 \times$ $2 \times 2 \mathrm{~mm}^{3}$ voxels, and spatially smoothed with a Gaussian kernel of $8 \mathrm{~mm}$ full-width at half-maximum.

To avoid brain activity related to uncertainty, which might include activity unrelated to memory retrieval, we selected only trials with high confidence (very sure old responses) for correct picture recognition (hit trials). We included only very sure hit trials also because prior research has shown that emotion has a larger impact on memory for items recalled with high confidence or with more vividness (e.g., Dolcos et al., 2005; Talarico et al. 2004). Trials were assigned to the Neutral or Arousing Encoded context condition based on each individual's subjective judgments to the sounds during the second run of the encoding session as either arousing or neutral.

Statistical analyses were performed within the framework of the general linear model (GLM: Friston et al., 1995). Two separate models were constructed for observing brain activity during successful picture recognition. One model tested how Encoded context (Arousing/Neutral) influences the retrieval of neutral content with Time (Recent/Remote). A second model explored the interaction between context at encoding and retrieval, by grouping the data according to Encoded context (Arousing/Neutral) and Retrieved context (Arousing/Neutral).

For the first model (factors: Encoded context and Time), trials were sorted into the following conditions: 1) remote very sure picture hits encoded under arousing context (Remote Hit E-Arousing), 2) remote very sure picture hits encoded under neutral context (Remote Hit E-Neutral), 3) recent very sure picture hits encoded under arousing context (Recent Hit E-Arousing), 4) recent very sure picture hits encoded under neutral context ( $\mathrm{Re}$ cent Hit E-Neutral, 5) all unsure picture hits from both remote and recent condition, 6) all remote and recent misses, 7) correct rejections, 8) false alarms, 9) other trials (filler trials and pictures of which the paired sound was not rated on arousal at encoding), and 10) null events.

For the second model (factors: Encoded and Retrieved contexts), the trials were sorted according to participants' response to the encoded and retrieved sound (context): 1) very sure picture hits with correct recall of the arousing sound context (Encoded context Arousing and Retrieved as Arousing: E-Arousing \& R-Arousing), 2) very sure picture hits with incorrect recall of the sound context as neutral (Encoded context Arousing but Retrieved as Neutral: E-Arousing \& R-Neutral), 3) very sure picture hits with correct recall of the neutral sound context (Encoded context Neutral and Retrieved as Neutral: E-Neutral \& R-Neutral), 4) very sure picture hits with incorrect recall of the sound context as arousing (Encoded context Neutral but Retrieved as Arousing: E-Neutral \& R-Arousing), 5) unsure picture hits, 6) picture misses, 7) correct rejection pictures, 8) false alarms pictures, 9) other trials (filler trials and pictures of which the paired sound was not rated at encoding), and 10) null events.

For both models, the 10 explanatory variables, one for each condition, were included in the model. These explanatory variables were temporally convolved with the canonical hemodynamic response function (HRF) and its temporal derivative. Each picture onset was modeled by a stick function. The duration of the null events was set to $10 \mathrm{~s}$. Both of the design matrices included the six head motion regressors (three translations, three rotations). A high pass filter was implemented using a cut-off period of $128 \mathrm{~s}$ to remove low-frequency effects from the time series. For statistical analysis, relevant contrast parameter images were generated for each participant and subsequently subjected to secondlevel analyses (Penny et al., 2003) treating participants as a random variable.

Contrasts for each of the four hit conditions against the baseline condition (null events) for each participant were subjected to a second level analysis in the factorial design. Furthermore, the four hit against correct rejection contrasts were also tested in the factorial design for the first model. Results of all second level analyses were initially thresholded at $p<.001$ (voxel-level uncorrected). For the whole-brain search, further cluster-size statistics were used as the test statistics, applying a threshold of $p_{F W E}<.05$, family-wise error corrected for multiple comparisons (Hayasaka and Nichols, 2003). The local maxima of significant clusters are reported in MNI coordinates. As we were interested specifically in the involvement of the hippocampus and the amygdala, for specific contrasts of interest, we applied a small volume correction (SVC) on the region of interest (ROI) using an anatomical mask including the bilateral the hippocampi and the amygdalae or when the hippocampus was the main region of interest, an anatomical mask of bilateral hippocampi, according to the AAL template (Tzourio-Mazoyer et al., 2002) provided by WFU PickAtlas software (Maldjian et al., 2003). For these contrasts, an initial voxel level threshold of $p<.001$ and a cluster-size statistics $p_{F W E}<.05$ within the ROI was applied.

We applied functional connectivity analyses to investigate which brain areas co-activate when cued with the picture stimulus. Since all memory retrieval trials were prompted with landscape pictures, we first created a bilateral ROI in a part of the parahippocampal gyrus, an area known to be involved in processing of scenes (Epstein et al., 1999), that was active when observing landscape photos during the localiser task. A conjunction of the anatomical parahippocampal gyri defined by the AAL template (Maldjian et al., 2003; Tzourio-Mazoyer et al., 2002) and the functional activation map on the group level for the contrast landscape $>$ faces of the localiser task mentioned below served as the seed ROI used in the psycho-physiological interaction analyses (PPI). For every participant, PPI were run for two models. The first model contrasted two categories of psychological factors: 1) 
Remote against Recent for all very sure hit trials, and 2) Encoded context Arousing against Neutral for both the Remote and Recent trials pooled. The second model contrasted two categories as well: 1) Encoded context Arousing (E-Arousing) against Neutral (ENeutral) for retrieved contexts pooled (R-Arousing + R-Neutral), and 2) context retrieved as Arousing (R-Arousing) against Neutral (R-Neutral) for both encoded contexts pooled (E-Arousing + ENeutral). For each model, two contrast images per participant, one for each category of contrast, were then subjected to second level one sample $t$-tests.

The localiser task was modeled with box-car functions with landscapes, faces, and scrambles as conditions of interest, and the contrast between landscapes and faces for each participant was subjected to the second-level one sample t-test. Voxel level $p_{F W E}<.05$ was applied as statistical threshold for this comparison. Significant clusters within the bilateral parahippocampal gyri defined by the AAL template were taken as parahippocampal seed ROI for PPI analysis described above.

\section{Results}

\subsection{Sound context manipulations}

\subsubsection{Sound rating}

Participants' ratings of arousal level of the sounds (six-point Likert scales, $1=$ calm, $6=$ arousing) during sound rating confirmed our emotional context manipulation (arousing sounds: $M \pm S D$ $5.2 \pm 1.0$; neutral sounds: $M \pm S D 2.5 \pm 1.2$ ). Ratings were in concordance with previous reports (Bradley and Lang (2007)), and did not differ between the recent and remote sessions $\left(F_{(1,23)}=0.156\right.$, $p=.696, \quad \eta^{2}=.007$, arousing: $\mathrm{t}_{(23)}=0.584, \quad p=.565$, neutral: $\left.\mathrm{t}_{(23)}=0.128, p=.899\right)$.

\subsubsection{Context rating at encoding}

Participants' responses to the sound question during the picture-sound encoding sessions were also in line with the Bradley and Lang's rating. The total number of responses as arousing or neutral were not significantly different from each other (remote: $t_{(23)}=-0.121, p=.905$, recent: $t_{(23)}=0.632, p=.533$ ), and did not change between sessions (arousing: $t_{(23)}=-1.309, p=.204$, neutral: $t_{(23)}=1.097, p=.284$ ) showing that participants responded to an equal number of contexts as being either arousing or neutral in both sessions (Table 1), indicating that there was no overall response bias at encoding.

\subsubsection{Possible response bias of context as arousing or neutral at retrieval}

To test whether there was a response bias towards endorsing the sound context to being either arousing or neutral at retrieval, the number of responses to the sound question given as arousing or neutral were compared. When all trials were included, there was no significant difference between the number of responses as being arousing or neutral $(M \pm S E$ : R-Arousing $74.9 \pm 4.6$, R-Neutral $\left.64.0 \pm 4.4, t_{(23)}=1.66, p=.111\right)$. However, when only trials whose picture was responded as definitely old were tested (including the false alarm trials), there were more arousing responses than neutral responses $(M \pm S E$ : Arousing $51.5 \pm 4.7$, Neutral $40.0 \pm 4.1$, $\left.t_{(23)}=2.23, p=.04\right)$.

\subsection{Content (picture) memory performance (Encoded context $\times$ Time)}

First we were interested in the effect of the context at encoding (E-Arousing/E-Neural) after a short or long delay (Recent/Remote) on memory performance for neutral content (pictures) and the

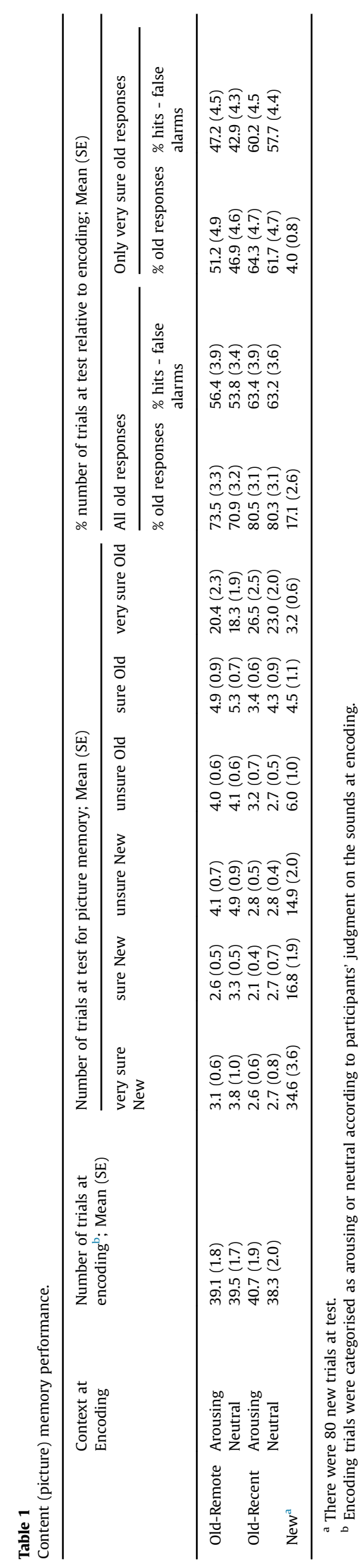


A) Content (picture) memory

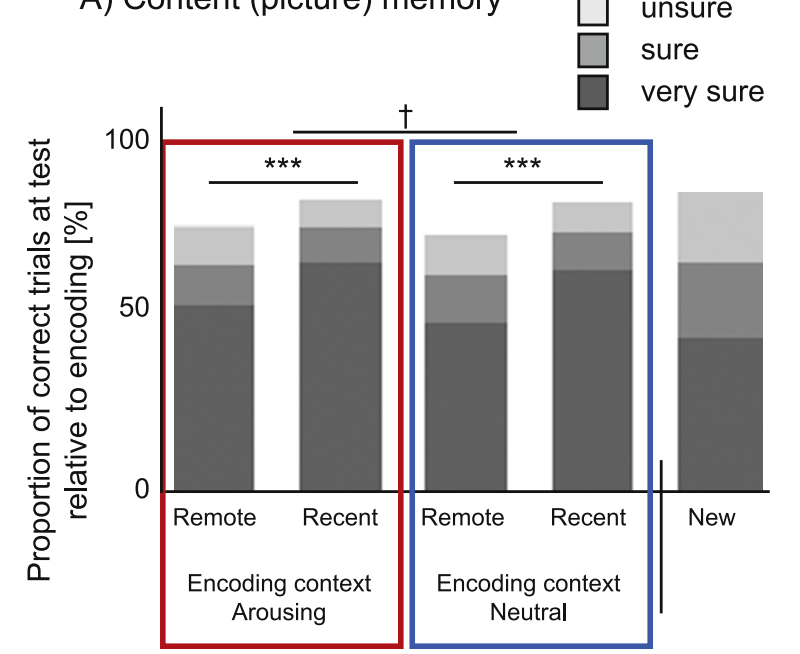

B) Context (sound) memory for pictures recognised with high confidence

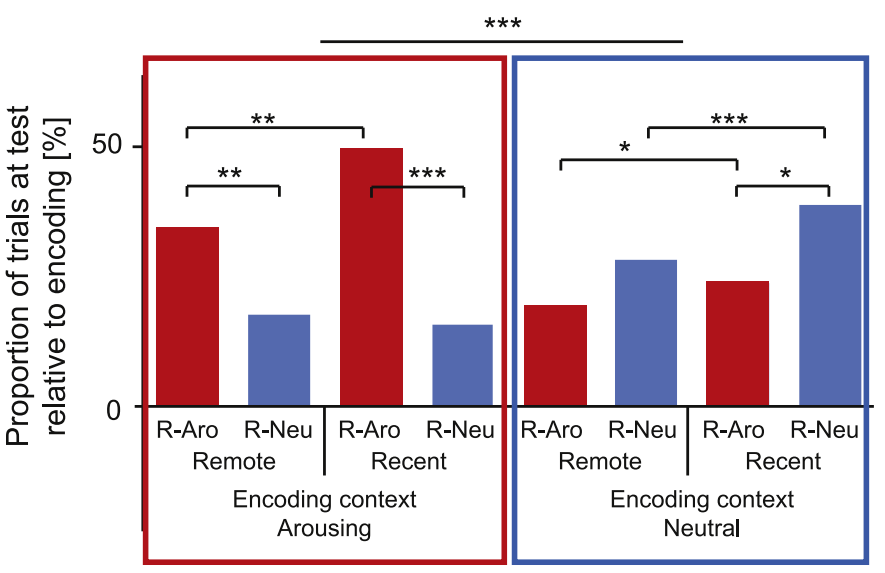

- Retrieved as Arousing

- Retrieved as Neutral

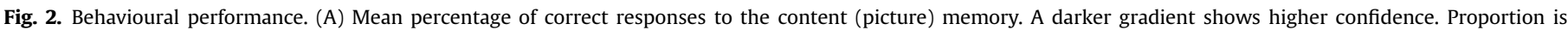

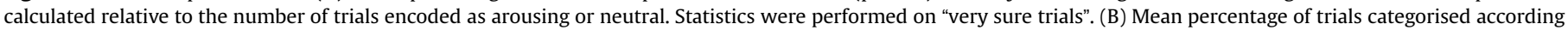

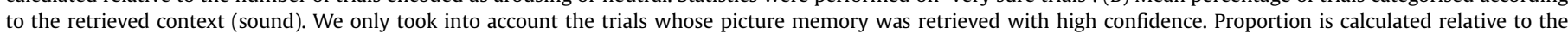
number of trials encoded as arousing or neutral. R-Aro: retrieved context arousing, R-Neu: retrieved context neutral. ${ }^{* * *} \mathrm{p}<.001$, ${ }^{* *} \mathrm{p}<.01$, ${ }^{*} \mathrm{p}<.05$, $+\mathrm{p}<.1$.

neural responses during recognition of the pictures.

\subsubsection{Behavioural results}

The behavioural performance scores during the recognition test can be found in Table 1 and Fig. 2A. Prior research has shown that an emotional effect on memory is most prominent for the items that are recognised with high confidence or recalled vividly (e.g., Dolcos et al., 2005; Talarico et al., 2004). Thus we limited the analyses to pictures remembered with very high confidence and calculated the picture memory score for each Encoded context, for Remote and Recent conditions. The memory score was corrected for sensitivity by subtracting the very sure false alarm rates from the very sure hit rates. A repeated measures analysis of variance (ANOVA) on this score with the factors Encoded context (EArousing/E-Neutral) and Time (Recent/Remote) was performed. This revealed a main effect of Time $\left(F_{(1,23)}=23.35, p<.001\right.$, $\left.\eta^{2}=.504\right)$, a trend of Encoded context $\left(F_{(1,23)}=3.31, p=.082\right.$, $\left.\eta^{2}=.126\right)$, but no significant interaction. The memory for content (pictures) decayed over time. There was a trend for more pictures to be remembered if encoded in an arousing context. We also compared the memory performance when responses from all confidence levels were included. We observed a similar pattern, however, only the main effect of Time survived the significance threshold $\left(F_{(1,23)}=12.06, p=.002, \eta^{2}=.344\right)$.

\subsubsection{Brain activation pattern}

To investigate the neural responses to recognition of neutral content (pictures) influenced by the context at encoding (E-Arousing/ E-Neural) after a short and long delay (Recent/Remote), we compared the brain activation pattern for correctly recognised picture trials with very high confidence with factors Encoded context (E-Arousing/ E-Neutral) and Time (Recent/Remote). We found a main effect of Encoded context that showed a greater activation pattern for arousing context (E-Arousing (Remote + Recent) $>$ E-Neutral (Remote + Recent)) in the left postcentral gyrus (local maximum [ $-22-4458$ ], cluster size 1387 voxels, $p<.001$ ), paracentral to middle part of the cingulate cortex (local maximum [ $-6-1856$ ], cluster size 477 voxels, $p=.001$ ), and a cluster extending to left rolandic operculum (local maximum [ $-54-2$ 10], cluster size 209 voxels, $p=.042$ ). An ROI analysis using the bilateral hippocampus + amygdala mask revealed a significant main effect of Encoded context (E-Arousing $>$ E-Neutral) in the left mid-hippocampus (local maximum [ $-32-22-14]$, cluster size 50 voxels, $p_{\text {SVC-hippocampu }+ \text { amygdala }}=.031$ ]; Fig. 3 , Table 2 ). We did not observe a main effect of Time nor did we find significant clusters for an interaction effect. The pattern did not change when we used the hit > correct rejection contrasts for all conditions for each of the comparisons mentioned above, except for one structure in the left precuneus that became significant for the contrast Recent $>$ Remote (local maximum [ $-6-7040]$, cluster size 233 , voxels, $p=.025$ ). To increase confidence in our findings, we also compared the pattern of activation when correctly responded "sure" picture hit items were included in the above contrasts. This also yielded a similar pattern of results, albeit less significant voxels in each cluster. Similar to the hits $>$ correct rejection comparison, a significant cluster in the bilateral precuneus was observed for the main effect of Time (Recent $>$ Remote; local maxima [8 -68 38] [ $-6-72$ 38], cluster size 676, voxels, $p<.001$ ).

\subsubsection{Functional connectivity with the scene picture processing area in the parahippocampal gyrus}

Although we did not find a main effect of Time or an interaction effect in the activation pattern, systems consolidation has been observed to involve changes in functional connectivity between brain regions (Takashima, 2009). We therefore asked if the effect of Time could be captured by changes in functional connectivity. The trials were always probed with a picture of a scene. We thus investigated whether areas differently co-activated with the scene processing area in the parahippocampal seed region, defined using the functional cluster from the localiser task, for different conditions.

We were specifically interested in investigating if there was a change in the involvement of the hippocampus. The Recent $>$ Remote contrast showed a trend for the connectivity with the left mid-hippocampus (local maximum [ $-32-22-16]$, cluster size 13 voxels, $p_{\text {SVC-hippocampus }}=.088$, Fig. $4 \mathrm{~A}$ ) when analysis was restricted to the bilateral hippocampal ROI. This trend appears to show that the functional connectivity between the parahippocampal seed region and the hippocampus decreased with time. A comparison between Encoding contexts, E-Arousing versus 

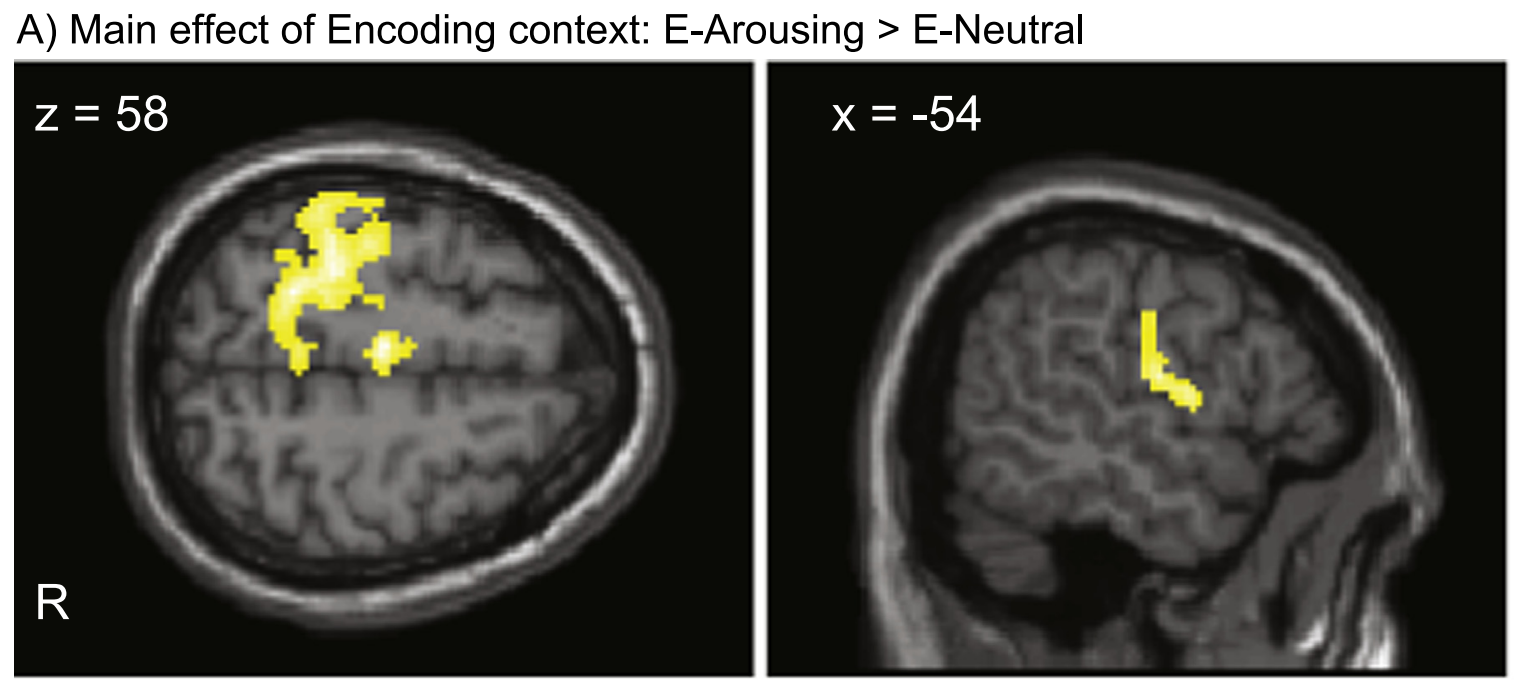

B) Hippocampal cluster for Encoding context: E-Arousing > E-Neutral
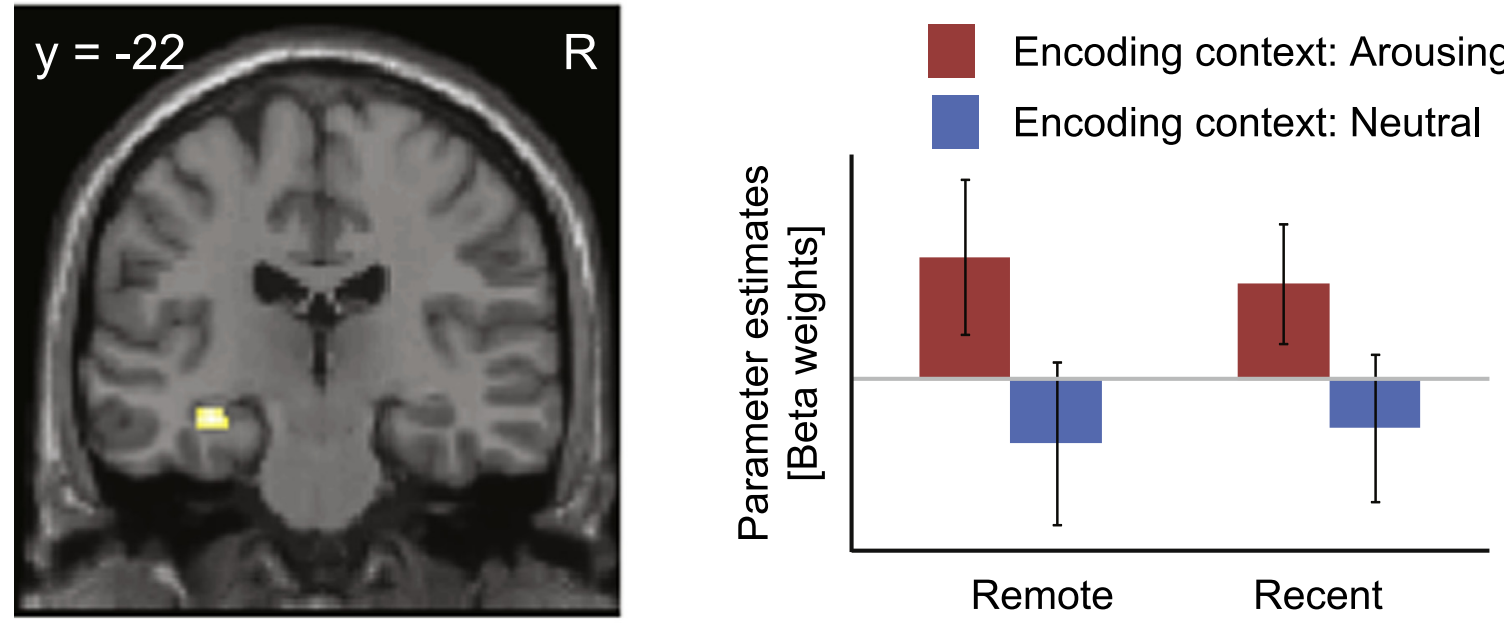

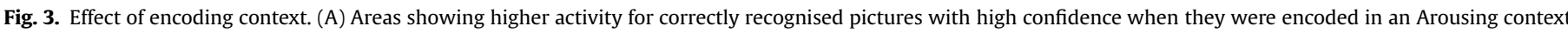

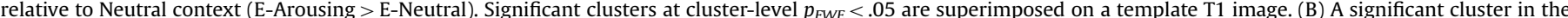

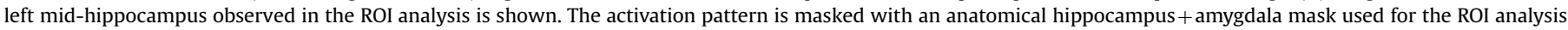

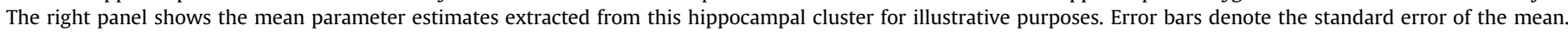

E-Neutral, did not show any significant effects.

\subsection{Effect of context memory on content memory performance (Encoded context $\times$ Retrieved context)}

Second, we investigated whether retrieved memory of neutral content (picture) is modulated by the context (sound) both at encoding and at retrieval.

\subsubsection{Behavioural results}

To test the influence of context both at encoding and retrieval, we re-categorised the conditions with two factors: Encoded context (EArousing/E-Neutral), and Retrieved context (R-Arousing/R-Neutral). Again, we only took into account the trials whose pictures were responded to as "old" with high confidence (Table 3, Fig. 2B). The performance score was calculated as the proportion of trials at test (R-Arousing, R-Neutral) relative to the number of trials at encoding separately for the two encoded conditions (E-Arousing, E-Neutral). For example, R-Arousing trials whose Encoded context was E-Arousing would be calculated as "number of trials encoded as arousing and responded as arousing" divided by "number of trials encoded as arousing". We corrected the performance score for sensitivity by subtracting the proportion of R-Arousing and R-Neutral responses made to the New pictures that were judged as "very sure old". A repeated measures ANOVA with the factors Encoded context (E-Arousing/E-Neutral), and Retrieved context (R-Arousing/R-Neutral) was performed on the corrected performance scores. We observed a main effect of Encoded context $(M \pm S E$ \%: E-Arousing $27.0 \pm 2.1$, E-Neutral $\left.19.8 \pm 1.6 ; F_{(1,23)}=28.727, p<.001, \eta^{2}=.555\right)$, Retrieved context $(M \pm S E$ \%: R-Arousing $29.1 \pm 2.4$, R-Neutral $\left.17.7 \pm 1.4 ; F_{(1,23)}=29.662, p<.001, \eta^{2}=.563\right)$ as well as an interaction effect $\left(F_{(1,23)}=29.418, p<.001, \eta^{2}=.561\right)$. Post-hoc $t$-tests (corrected $\alpha=.0125$ ) revealed that for the R-Arousing responses, there were more trials that were encoded under an arousing context $(M \pm S E \%$ : E-Arousing and R-Arousing $39.4 \pm 3.8$, E-Neutral but R-Arousing $\left.18.8 \pm 1.8 ; t_{(23)}=-.838, p=.411 ; t_{23}=5.895, p<.001\right)$. The R-Neutral responses were also generally in accordance with the encoded context $(M \pm S E$ \%: E-Neutral and R-Neutral $20.7 \pm 2.0$, E-Arousing but R-Neutral $\left.14.6 \pm 1.4 ; t_{(23)}=3.223, p=.004\right)$. For the Encoded context Arousing trials, retrieved context was more likely to be responded as Arousing $\left(t_{(23)}=6.207, p<.001\right)$, whereas for the Encoded context Neutral trials, retrieved context as Arousing or Neutral did not differ from each other $\left(t_{(23)}=.853, p=.411\right)$. Thus, overall, context retrieval matched to those at encoding, however, this was more so for the arousing context than for the neutral context. 
Table 2

Effect of encoding context

\begin{tabular}{|c|c|c|c|c|c|c|c|}
\hline \multicolumn{8}{|c|}{ Encoding context: E-Arousing $>$ E-Neutral } \\
\hline \multicolumn{2}{|c|}{ cluster } & \multicolumn{5}{|l|}{ peak } & \multirow[t]{2}{*}{ Region } \\
\hline \multicolumn{7}{|c|}{ MNI coordinates } & \\
\hline size & $P_{F W E}$ & $\mathrm{~T}$ & Z & $\mathrm{x}$ & $\mathrm{y}$ & $\mathrm{z}$ & \\
\hline \multirow[t]{5}{*}{1387} & $<.001$ & 4.75 & 4.43 & -22 & -44 & 58 & L postcentral gyrus \\
\hline & & 4.43 & 4.17 & -24 & -20 & 62 & L precentral gyrus \\
\hline & & 4.3 & 4.05 & -36 & -44 & 64 & L superior parietal lobule \\
\hline & & 4.14 & 3.92 & -16 & -46 & 60 & L precuneus \\
\hline & & 3.49 & 3.35 & -32 & -44 & 56 & L inferior parietal lobule \\
\hline \multirow[t]{5}{*}{477} & .001 & 4.71 & 4.4 & -6 & -18 & 56 & L paracentral lobule \\
\hline & & 4.03 & 3.82 & -8 & -16 & 44 & L middle cingulum \\
\hline & & 3.93 & 3.74 & 0 & -20 & 64 & $\begin{array}{l}\text { R supplementary motor } \\
\text { area }\end{array}$ \\
\hline & & 3.89 & 3.7 & 4 & -24 & 68 & R paracentral lobule \\
\hline & & 3.61 & 3.46 & -8 & -4 & 64 & $\begin{array}{l}\text { L supplementary motor } \\
\text { area }\end{array}$ \\
\hline \multirow[t]{2}{*}{209} & .042 & 4.38 & 4.12 & -52 & -12 & 16 & L postcentral gyrus \\
\hline & & 4.12 & 3.9 & -54 & -2 & 10 & L Rolandic operculum \\
\hline 50 & $.031^{\mathrm{a}}$ & 4.21 & 3.98 & -32 & -22 & -14 & L hippocampus \\
\hline \multicolumn{8}{|c|}{$\begin{array}{c}\text { Encoding context: E-Neutral }>\text { E } \\
\text { No significant clusters }\end{array}$} \\
\hline
\end{tabular}

${ }^{\text {a }} P_{F W E-S V C}$ on hippocampus + amygdala ROI, FWE: family-wise error corrected, L: left, R: right.

\subsubsection{Brain activation pattern}

To probe differences in neural activity related to context at encoding and at retrieval, we tested the activation pattern difference with two factors: Encoded context (E-arousing/E-neutral), and Retrieved context (R-arousing/R-neutral). Similar to the behavioural data comparison, the trials were categorised according to the participants' response to the context memory (sound) at encoding and at retrieval, resulting in the following conditions for all pictures remembered with high confidence: 1) E-Arousing and R-Arousing, 2) E-Arousing but R-Neutral, 3) E-Neutral and R-Neutral, and 4) E-Neutral but R-Arousing.

We found a main effect of Encoded context in the left precuneus (cluster size 399 voxels, local maximum [-14 - 46 58]) exhibiting greater responses when the context at encoding had been arousing compared to neutral (contrast E-Arousing (RArousing $+\mathrm{R}$-Neutral) $>$ E-Neutral (R-Arousing $+\mathrm{R}$-Neutral)). The reverse contrast (E-Neutral (R-Arousing $+\mathrm{R}$-Neutral) $>$ E-Arousing (R-Arousing + R-Neutral)) did not show any significant clusters.

A main effect of Retrieved context revealed that when participants indicated that the context had been arousing compared to neutral at retrieval, regardless of the veridical encoding context (R-Arousing (EArousing +E-Neutral) $>$ R-Neutral (E-Arousing +E-Neutral)), clusters in the left postcentral gyrus (cluster size 1458 voxels, local maximum [ $-36-2252], p<.001$ ) and the right lingual gyrus (cluster size 444 voxels, local maximum [12 - 742$], p=.001$ ) were more active. An ROI analysis also revealed a similar effect within the bilateral hippocampus + amygdala mask, with a significant cluster in the right anterior hippocampus (cluster size 66 voxels, local maximum [32 - 12 $-16], p_{\text {SVC-hippocampus }+ \text { amygdala }}=.020$ ) (Fig. 5), and the left hippocampus at trend (anterior cluster size 31 voxels, local maxima [ $-32-8-22]$, $p_{\text {SVC-hippocampus }+ \text { amygdala }}=.054$; posterior cluster size 32 local maximum

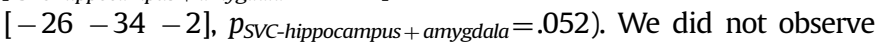
interaction effects.

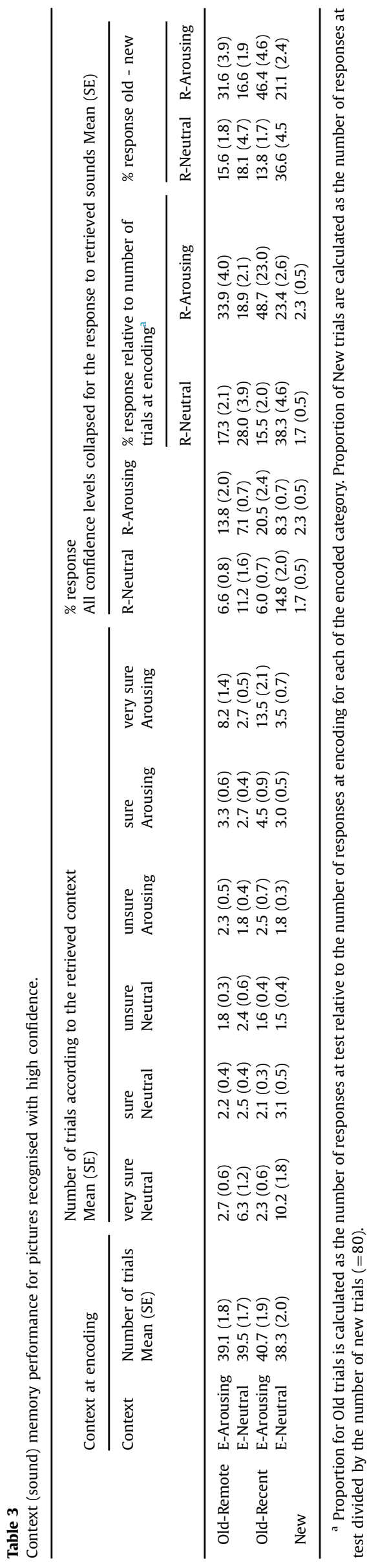


A) Recent $>$ Remote (trend)

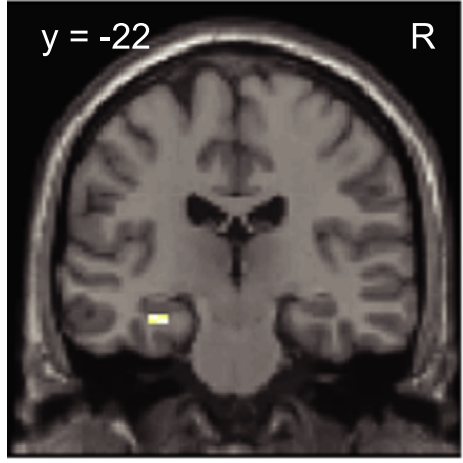

B) Retrieved context Arousing $>$ Neutral (significant clusters)

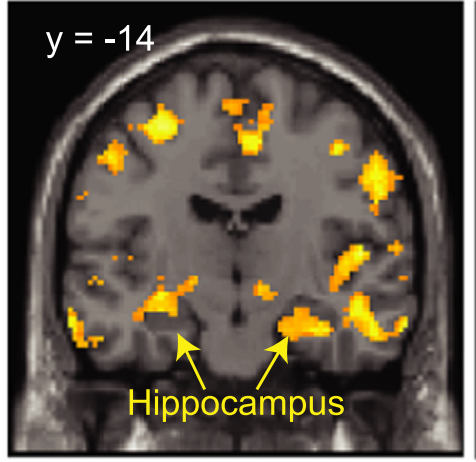

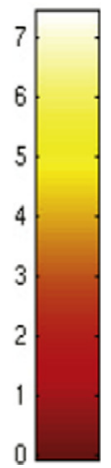

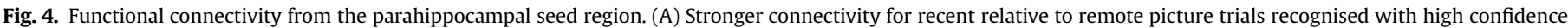

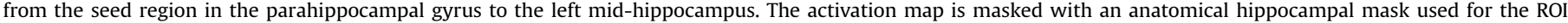

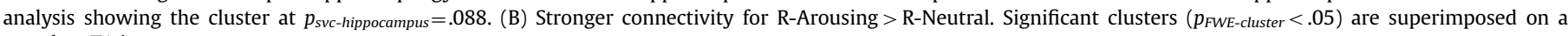
template T1 image.

Effect of retrieved context - activation

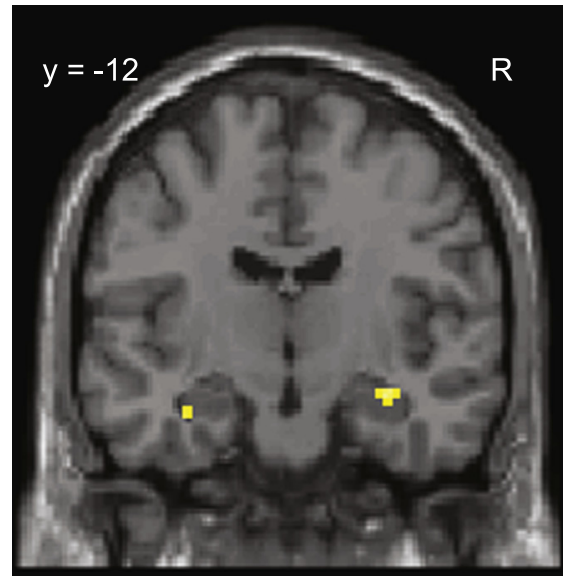

Encoding context: Arousing

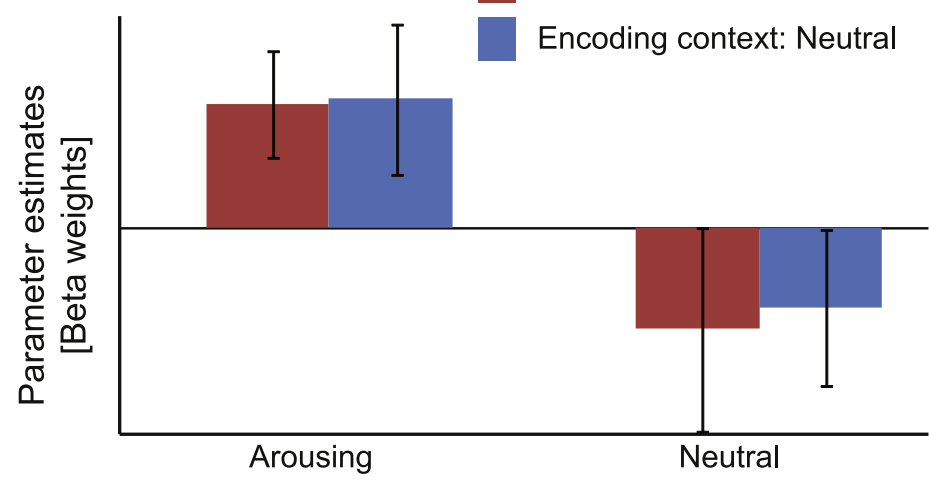

Retrieved context

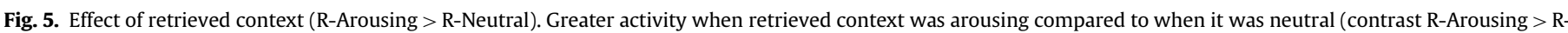

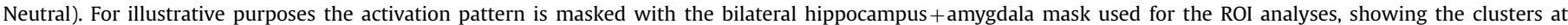

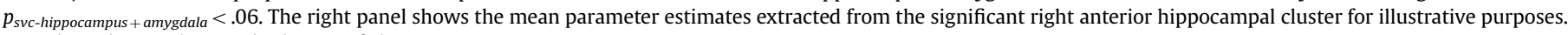
Error bars denote the standard error of the mean.

\subsubsection{Functional connectivity with the scene picture processing area in the parahippocampal gyrus}

Next we asked whether retrieving an arousing context versus a neutral context affected functional connectivity patterns with the parahippocampal region that was involved in processing scene information, i.e. a region critical to processing the neutral content in our memory task. Compared to retrieving a neutral context, when participants retrieved an arousing context, we observed greater functional connectivity between the parahippocampal ROI and the bilateral anterior hippocampus extending to the amygdala, and between the parahippocampal ROI and various neocortical areas (Fig. 4B, Table 4). The reverse contrast (R-neutral $>\mathrm{R}$ arousing) showed no significant clusters.

\section{Discussion}

Here we aimed to study both the behavioural and neural consequences of 1) an arousing context at encoding on neutral content memory observed during a retrieval test that may change as a function of study-test delay, and 2) whether there is an influence of retrieved context on recognition memory of neutral content. We predicted that an arousing context at encoding as compared to a neutral context would enhance content memory. Furthermore, we expected the hippocampus involvement at retrieval to decrease over time for content with a neutrally encoded context, but less so if the encoded context had been arousing. As for the influence of the retrieved context, we did not have a clear prediction as prior research has not focused on the emotional memory enhancement related to retrieved context.

Our behavioural results showed that even though the memory overall decreased over time, there was a trend for pictures encoded under arousing contexts to be remembered better. This pattern did not change as a function of time. The relationship between retrieved context and content memory revealed that participants were more likely to think the encoded context had been arousing for confidently remembered pictures, irrespective of the veridical encoded context. The overall number of responses to the sound question as being arousing or neutral was not significantly different. Thus, this effect was not driven by a response bias to the context question at retrieval. This identifies a relationship between content memory performance and the subjective emotional experience at the time of retrieval.

Our imaging results revealed that the enhancement of memory by an arousing context at encoding was accompanied by increased hippocampal responses at retrieval. A trend towards a decrease in functional connectivity between the scene processing area (the parahippocampal gyrus) and the mid-hippocampus with time was 
Table 4

Functional connectivity from the parahippocampal cortex.

\begin{tabular}{|c|c|c|c|c|c|c|c|}
\hline \multicolumn{8}{|c|}{ Retrieved context: $R$-Arousing $>$ R-Neutral } \\
\hline \multicolumn{2}{|c|}{ Cluster } & \multicolumn{5}{|l|}{ Peak } & \multirow[t]{2}{*}{ Region } \\
\hline \multicolumn{7}{|c|}{ MNI coordinates } & \\
\hline Size & $P_{F W E}$ & $\mathrm{~T}$ & $\mathrm{Z}$ & $\mathrm{x}$ & $\mathrm{y}$ & $\mathrm{z}$ & \\
\hline 1170 & $<.001$ & 7.08 & 4.89 & -38 & -54 & -32 & L cerebellum \\
\hline \multirow[t]{2}{*}{428} & $<.001$ & 7.3 & 4.98 & 0 & -6 & 58 & $\begin{array}{l}\text { L supplementary motor } \\
\text { area }\end{array}$ \\
\hline & & 6.4 & 4.62 & 6 & -10 & 54 & $\begin{array}{l}\text { R supplementary motor } \\
\text { area }\end{array}$ \\
\hline \multirow[t]{10}{*}{4552} & $<.001$ & 7.3 & 4.98 & -6 & -44 & 50 & L middle cingulum \\
\hline & & 7.04 & 4.88 & -34 & -12 & 58 & L precentral gyrus \\
\hline & & 5.41 & 4.16 & -58 & -24 & 36 & L supramarginal gyrus \\
\hline & & 5.35 & 4.13 & -30 & -32 & 56 & L postcentral gyrus \\
\hline & & 5.34 & 4.12 & -16 & -44 & 60 & L precuneus \\
\hline & & 5.25 & 4.08 & -12 & -44 & 34 & L middle cingulum \\
\hline & & 6.42 & 4.63 & 48 & -42 & 48 & $\mathrm{R}$ inferior parietal lobule \\
\hline & & 6.01 & 4.44 & 44 & -22 & 54 & R postcentral gyrus \\
\hline & & 5.81 & 4.35 & 54 & -36 & 44 & R supramarginal gyrus \\
\hline & & 5.43 & 4.17 & 18 & -46 & 60 & $\mathrm{R}$ superior parietal lobule \\
\hline 228 & .006 & 6.7 & 4.74 & -28 & 30 & 40 & L middle frontal gyrus \\
\hline \multirow[t]{5}{*}{4182} & $<.001$ & 6.66 & 4.73 & 52 & -32 & -12 & $\mathrm{R}$ middle temporal gyrus \\
\hline & & 6.52 & 4.67 & 50 & -12 & 8 & $\mathrm{R}$ superior temporal gyrus \\
\hline & & 6.11 & 4.49 & 32 & -12 & -22 & R hippocampus \\
\hline & & 5.95 & 4.42 & 22 & -74 & -30 & $\mathrm{R}$ cerebellum \\
\hline & & 5.46 & 4.18 & 56 & -18 & -24 & $\mathrm{R}$ inferior temporal gyrus \\
\hline \multirow[t]{2}{*}{257} & .003 & 6.63 & 4.72 & 56 & -12 & 28 & R supramarginal gyrus \\
\hline & & 5.52 & 4.21 & 58 & -14 & 32 & $\mathrm{R}$ postcentral gyrus \\
\hline \multirow[t]{2}{*}{320} & .001 & 6.56 & 4.69 & -52 & -4 & -24 & L middle temporal gyrus \\
\hline & & 5.57 & 4.24 & -66 & -24 & -22 & L inferior temporal gyrus \\
\hline \multirow[t]{6}{*}{1863} & $<.001$ & 6.43 & 4.63 & -34 & 14 & 6 & L insula \\
\hline & & 5.66 & 4.28 & 14 & 24 & -4 & R caudate \\
\hline & & 5.39 & 4.15 & 16 & 62 & 6 & R superior frontal gyrus \\
\hline & & 5.36 & 4.13 & -60 & 6 & 10 & $\begin{array}{l}\text { L inferior frontal gyrus } \\
\text { pars opercularis }\end{array}$ \\
\hline & & 5.33 & 4.12 & -58 & 8 & 6 & L Rolandic operculum \\
\hline & & 5.03 & 3.96 & -58 & -4 & 16 & L postcentral gyrus \\
\hline \multirow[t]{3}{*}{587} & $<.001$ & 6.17 & 4.52 & -34 & -20 & -12 & L hippocampus \\
\hline & & 5.02 & 3.96 & -28 & -10 & -2 & L putamen \\
\hline & & 4.92 & 3.9 & -28 & -36 & -12 & L parahippocampal gyrus \\
\hline \multirow[t]{5}{*}{821} & $<.001$ & 6.05 & 4.46 & -56 & -20 & 4 & L superior temporal gyrus \\
\hline & & 5.87 & 4.38 & -54 & -18 & 0 & L middle temporal gyrus \\
\hline & & 5.5 & 4.2 & -52 & -42 & -14 & $\mathrm{~L}$ inferior temporal gyrus \\
\hline & & 4.89 & 3.88 & -42 & -20 & 6 & L Heschl's gyrus \\
\hline & & 4.43 & 3.63 & -66 & -24 & 18 & L supramarginal gyrus \\
\hline \multirow[t]{2}{*}{614} & $<.001$ & 5.35 & 4.13 & -40 & -68 & 44 & $\mathrm{~L}$ angular gyrus \\
\hline & & 4.71 & 3.78 & -50 & -44 & 40 & $\mathrm{~L}$ inferior parietal lobule \\
\hline 209 & .01 & 5.2 & 4.05 & -44 & -60 & 22 & L middle temporal gyrus \\
\hline \multirow[t]{2}{*}{155} & .037 & 4.83 & 3.85 & -14 & 50 & -16 & L orbito-frontal gyrus \\
\hline & & 4.55 & 3.7 & -4 & 46 & -20 & $\mathrm{~L}$ rectus \\
\hline \multicolumn{8}{|c|}{$\begin{array}{l}\text { Retrieved context: } \text { R-Neutral }>\text { R-Arousing } \\
\text { No significant clusters }\end{array}$} \\
\hline \multicolumn{8}{|c|}{ Encoding context: E-Arousing $>$ E-Neutral } \\
\hline \multicolumn{8}{|c|}{ No significant clusters } \\
\hline \multicolumn{8}{|c|}{$\begin{array}{l}\text { Encoding context: } \text { E-Neutral }>\text { E-Arousing } \\
\quad \text { No significant clusters }\end{array}$} \\
\hline
\end{tabular}

L: left, R: right, FWE: family-wise error corrected.

observed, whereas we did not find evidence for a change in net hippocampal activation levels between recently and remotely encoded items. Just as in the behavioural data, retrieved context had an impact on the neural pattern at retrieval. More specifically, if subjects thought the context had been arousing, greater activation in the anterior hippocampus and increased functional connectivity between the hippocampus and the parahippocampal gyrus were detected.

\subsection{Memory enhancement by emotional context}

Behaviourally, we only observed a trend in the emotional context enhancement. Although enhancement of emotional memories is established behaviourally with possible neural mechanistic explanations (reviewed in e.g., Dolcos and Denkova (2014), LaBar and Cabeza (2006), McGaugh (2004), Phelps (2006)), there have been some discussions regarding the modulatory effect of emotional content/context on memory performance for associative/relative memories. Some studies show enhancement whereas others show impairment or no effect (reviewed in: Chiu et al. (2013), Mather and Sutherland (2011)). The review by Mather and Sutherland (2011) suggests that memory is enhanced if the emotional part of the encoded material draws attention to the features that are tested later on. Chiu et al. (2013) propose that memory enhancement is observed when the memory content is fused or unitised with the emotional aspect of the experience. In contrast, if the emotional aspect does not guide attention to, or if the emotionality is not fused with the representation to be tested, impairment is often reported. We tried to avoid an attentional bias to either the picture or the sound by having two rounds of encoding, once with the attention allocated to the non-emotional visual content, and then to the emotional aspect, that is, the sound information. Furthermore, we instructed the participants that both sound and picture information would be important for the memory test later. Even though the manipulation to associate arousing context to the neutral memory representations by instructing participants that remembering the picture-sound association is important for this experiment, the emotional enhancement effect was modest probably because the sound context was only mildly arousing. Deep encoding instruction in this study furthermore tampered the possible emotional memory enhancement effect, as this effect is known to be stronger under shallow than deep encoding situations (e.g., Strange et al., 2003). Despite these factors such as relatively mild aversive experience, deep encoding, habituation due to repetition, that might work against the enhancement effect of emotion on memory, our results are nonetheless in line with prior studies showing the emotional enhancement effect. On the neural level, we observed increased responses for pictures encoded under an arousing context in the mid-hippocampus, an area that is central to memory encoding and retrieval. Since our behavioural effect was modest, results should be interpreted with caution. Regardless, our results extend previous research that suggests the amygdala to up-regulate hippocampal processing during encoding, consolidation (McGaugh, 2006; Richardson et al., 2004), and retrieval (Dolcos et al., 2005; Ritchey et al., 2008; Sharot et al., 2007; Smith et al., 2005, 2006, 2004; Sterpenich et al., 2009) by indicating that arousing encoding contexts can result in increased hippocampal involvement at the time of retrieval.

The focus of our study was on the "arousal" rather than the "valence" axis of emotional context because the amygdala is known to react on salient information, thus more for arousing than non-arousing stimuli, which could be both positive and negative in valence (Bradley et al., 1992; McGaugh, 2013). For this reason, we also asked the participants whether they thought the sounds were "arousing" or not, rather than "pleasant/unpleasant" throughout the encoding and test phases. For stimulus selection limitations and to reduce possible confounding effects of valence, the sounds that we chose to be arousing were also unpleasant at the same time. However, it is also interesting to observe differential effect of valence (positive versus negative) in future studies (cf., Beyeler et al., 2016; Bradley et al., 1992). 


\subsection{Effects of retrieved context on neutral content memory}

The participants were more likely to attribute the associated sound context as arousing if the picture memory was remembered with high confidence. This finding cannot be explained by a response bias at retrieval, as the overall number of responses to the sound question as being arousing or neutral was not significantly different.

When we compared the brain activation pattern according to the retrieved context, we observed that the increased engagement of the hippocampus at the time of retrieval was not merely reflecting the veridical re-instantiation of the encoded context. Specifically, when participants were highly confident about remembering the neutral content (picture), they were more likely to think that the encoded context (sound) had been arousing, and when participants thought the encoded context had been arousing (R-arousing) we observed greater anterior hippocampal responses and a stronger functional connectivity between the parahippocampal gyrus and the hippocampus close to the junction with the amygdala. These results point to greater involvement of the hippocampus when the retrieved context was arousing irrespective of the actual encoded context. Memories of emotionally arousing events are often vivid, detailed, accompanied by a high feeling of confidence (Kensinger et al., 2011; Sharot et al., 2004, 2007; Todd et al., 2013), despite potential inaccuracy (Neisser and Harsch, 1992; Talarico and Rubin, 2003), and are associated with increased hippocampal involvement (for reviews: Cahill and McGaugh (1998), Christianson (1992), Diana et al. (2007a, 2007b), Kensinger and Corkin (2004), LaBar and Cabeza (2006), Phelps (2006)). Our results question cause-and-effect, and suggest that if one feels confident about memory retrieval one often assumes the experience must have been arousing. The neural data seem to be in favour of the interpretation that the anterior hippocampal activation increase was due to the emotional aspect of the memory that was retrieved, rather than due to the richness of the retrieved memory. We found differential hippocampal involvement for pictures remembered with confidence depending on whether the associated sound context was retrieved as arousing or neutral. One interpretation is that the retrieval of arousing context resulted in hippocampal activation. Alternatively, hippocampal activation might have caused a more vivid recollection, and as a result, participants may have inferred that the context was probably arousing. People are usually more confident of their response when the memory is more vivid and rich (Yonelinas et al., 1996) and the activation of the hippocampus is often reported to be high when the memory is vivid (e.g., Addis et al., 2004; Diana et al., 2007a, 2007b; Dolcos et al., 2005). Critically, we found increased hippocampal response when participants thought the sound was arousing compared to when they thought the sound was neutral, even though for both conditions we only included trials for which the picture memory was recognised with high confidence by the participants. Thus, the neural data seem to be mostly in line with the interpretation that greater anterior hippocampal activation was due to the arousing aspect of the memory that was retrieved, rather than due to the richness of the retrieved memory.

A point to mention is that the emotional context effect was found in the anterior part of the hippocampus, whereas the decrease in functional connectivity with time was found in the midhippocampus. Prior studies suggest a functional distinction between the anterior and posterior hippocampus (reviewed in Fanselow and Dong (2010), Strange et al. (2014)). The anterior part is involved more in emotional aspects, whereas the posterior part responds more to spatial/item related memories. The consolidation trajectory may differ within the hippocampus depending on the functional role the subregions subserve.

Memory retrieval is not only about accurately re-instantiating the past. It has been suggested that each time a memory is retrieved, it is subjected to another round of encoding (Dudai, 2012; Nadel and Moscovitch, 1997). We found increased activity and functional connectivity with the hippocampus close to the junction of the amygdala when subjects responded to the context question as arousing, which may reflect reconstruction and modification of the retrieved episode rather than merely pure retrieval related processes. One could speculate that amygdala-hippocampal coupling during recognition of neutral content has a strengthening effect on the retrieved memory trace by re-encoding it as an emotionally laden memory. Recent studies showed that blocking noradrenergic functioning during reactivation of memories affected the re-encoding or retrieval process, abolishing a subsequent emotional memory enhancement (Kroes et al., 2010; Schwabe et al., 2013). Since our observations were only partially within the amygdala structure, caution is warranted about the interpretation of cross-talk between the hippocampus and the amygdala for re-encoding of emotional contexts. Moreover, it is a challenge to dissociate brain responses that are retrieval related or re-encoding related during a recognition test. One study tried to dissociate the brain activation pattern for successful retrieval of emotional material from that of incidental encoding (Shafer and Dolcos, 2014) by having two retrieval tests, one testing the studied material (retrieval success), and another testing for the memory of the items used as lures during the first test (incidental encoding). Those authors report that the hippocampus and the parahippocampal structures were more involved in the retrieval of emotional memories, that is, the activation in these areas were high if the originally studied emotional material was recognised. The right amygdala, in contrast, was involved in both retrieval and encoding of the emotional stimulus, as this area showed heightened activation also for emotional lure items that were subsequently remembered. However, they still cannot differentiate reencoding from retrieval, as the reported activation pattern related to incidental encoding was observed for novel items and not for studied items. Future studies on memory of re-encoded material may give further insights in the neural processes that lead to changes in emotional memory retention. Nonetheless, our results provide an initial indication that emotional memories could evolve through retrieval of memories associated with an arousing context that may have consequences when recalled again at a later point in time.

\section{Conclusion}

Using a prospective memory paradigm associating neutral pictures to either an arousing or neutral sound context at encoding, we provide evidence that memory for neutral content can be affected by the arousing context and that the hippocampus is differentially involved depending on the retrieved context, exerting a heightened activation and functional connectivity with the areas processing the content if the retrieved context was arousing. These results reveal a critical role for arousal in governing memory flexibility over time and provide insight into the neural mechanisms underlying emotional memory consolidation and retrieval.

\section{Acknowledgement}

This study was supported by a grant from the Netherlands Organization for Scientific Research (NWO 451-06-006). The authors declare no competing financial interests. 


\section{References}

Addis, D.R., Moscovitch, M., Crawley, A.P., McAndrews, M.P., 2004. Recollective qualities modulate hippocampal activation during autobiographical memory retrieval. Hippocampus 14 (6), 752-762.

Alvarez, P., Squire, L.R., 1994. Memory consolidation and the medial temporal lobe: a simple network model. Proc. Natl. Acad. Sci. USA 91 (15), 7041-7045.

Beyeler, A., Namburi, P., Glober, Gordon, F., Simonnet, C., Calhoon, Gwendolyn, G., Conyers, Garrett, F., Luck, R., Wildes, Craig, P., Tye, Kay, M., 2016. Divergent routing of positive and negative information from the amygdala during memory retrieval. Neuron 90 (2), 348-361. http://dx.doi.org/10.1016/j.neuron.2016.03.004.

Bradley, M.M., Lang, P.J., 2007. International Affective Digitized Sounds (2nd Edition; IADS-2): Affective Rating of Sounds and Instruction Manual. The Center for Research in Psychophysiology, University of Florida, Gainesville, FL.

Bradley, M.M., Greenwald, M.K., Petry, M.C., Lang, P.J., 1992. Remembering pictures: pleasure and arousal in memory. J. Exp. Psychol.: Learn. Mem. Cogn. 18 (2), 379-390. http://dx.doi.org/10.1037/0278-7393.18.2.379.

Cahill, L., 2010. Sex influences on brain and emotional memory: the burden of proof has shifted. Prog. Brain Res. 186, 29-40. http://dx.doi.org/10.1016/ b978-0-444-53630-3.00003-8.

Cahill, L., McGaugh, J.L., 1998. Mechanisms of emotional arousal and lasting declarative memory. Trends Neurosci. 21 (7), 294-299. http://dx.doi.org/10.1016 s0166-2236(97)01214-9.

Chiu, Y.-C., Dolcos, F., Gonsalves, B.D., Cohen, N.J., 2013. On opposing effects of emotion on contextual or relational memory. Front. Psychol., 4. http://dx.doi. org/10.3389/fpsyg.2013.00103.

Christianson, S.-Å., 1992. Emotional stress and eyewitness memory: a critical review. Psychol. Bull. 112 (2), 284-309. http://dx.doi.org/10.1037/0033-2909.112.2.284.

Diana, R.A., Yonelinas, A.P., Ranganath, C., 2007a. Imaging recollection and familiarity in the medial temporal lobe: a three-component model. Trends Cogn. Sci 11 (9), 379-386.

Diana, R.A., Yonelinas, A.P., Ranganath, C., 2007b. Imaging recollection and familiarity in the medial temporal lobe: a three-component model. Trends Cogn. Sci. 11 (9), 379-386. http://dx.doi.org/10.1016/j.tics.2007.08.001.

Dolcos, F., Denkova, E., 2014. Current emotion research in cognitive neuroscience: linking enhancing and impairing effects of emotion on cognition. Emot. Rev. 6 (4), 362-375. http://dx.doi.org/10.1177/1754073914536449.

Dolcos, F., LaBar, K.S., Cabeza, R., 2005. Remembering one year later: role of the amygdala and the medial temporal lobe memory system in retrieving emotional memories. Proc Natl Acad Sci USA 102 (7), 2626-2631. http://dx.doi.org/ $10.1073 /$ pnas.0409848102.

Dudai, Y., 2012. The restless engram: consolidations never end. Annu. Rev. Neurosci. 35 (1), 227-247. http://dx.doi.org/10.1146/annurev-neuro-062111-150500.

Dunsmoor, J.E., Kragel, P.A., Martin, A., LaBar, K.S., 2014. Aversive learning modulates cortical representations of object categories. Cereb. Cortex 24 (11), 2859-2872. http://dx.doi.org/10.1093/cercor/bht138.

Dunsmoor, J.E., Murty, V.P., Davachi, L., Phelps, E.A., 2015. Emotional learning selectively and retroactively strengthens memories for related events. Nature 520 (7547), 345-348. http://dx.doi.org/10.1038/nature14106.

Epstein, R., Harris, A., Stanley, D., Kanwisher, N., 1999. The parahippocampal place area: recognition, navigation, or encoding? Neuron 23 (1), 115-125. http://dx doi.org/10.1016/s0896-6273(00)80758-8.

Fanselow, M.S., Dong, H.-W., 2010. Are the dorsal and ventral hippocampus functionally distinct structures? Neuron 65 (1), 7-19. http://dx.doi.org/10.1016/j. neuron.2009.11.031.

Friston, K.J., Frith, C.D., Turner, R., Frackowiak, R.S., 1995. Characterizing evoked hemodynamics with fMRI. Neuroimage 2 (2), 157-165.

Hayasaka, S., Nichols, T.E., 2003. Validating cluster size inference: random field and permutation methods. Neuroimage 20 (4), 2343-2356.

Kensinger, E.A., Corkin, S., 2004. Two routes to emotional memory: distinct neura processes for valence and arousal. Proc Natl Acad Sci USA 101 (9), 3310-3315. http://dx.doi.org/10.1073/pnas.0306408101.

Kensinger, E.A., Addis, D.R., Atapattu, R.K., 2011. Amygdala activity at encoding corresponds with memory vividness and with memory for select episodic details. Neuropsychologia 49 (4), 663-673. http://dx.doi.org/10.1016/j. neuropsychologia.2011.01.017.

Knight, D.C., Cheng, D.T., Smith, C.N., Stein, E.A., Helmstetter, F.J., 2004. Neural substrates mediating human delay and trace fear conditioning. J. Neurosci. 24 (1), 218-228. http://dx.doi.org/10.1523/jneurosci.0433-03.2004.

Kroes, M.C.W., Strange, B.A., Dolan, R.J., 2010. $\beta$-adrenergic blockade during memory retrieval in humans evokes a sustained reduction of declarative emotional memory enhancement. J. Neurosci. 30 (11), 3959-3963. http://dx doi.org/10.1523/jneurosci.5469-09.2010.

LaBar, K.S., Phelps, E.A., 1998. Arousal-mediated memory consolidation: role of the medial temporal lobe in humans. Psychol. Sci. 9 (6), 490-493. http://dx.doi.org/ 10.1111/1467-9280.00090.

LaBar, K.S., Cabeza, R., 2006. Cognitive neuroscience of emotional memory. Nat. Rev. Neurosci. 7 (1), 54-64. http://dx.doi.org/10.1038/nrn1825.

Maldjian, J.A., Laurienti, P.J., Kraft, R.A., Burdette, J.H., 2003. An automated method for neuroanatomic and cytoarchitectonic atlas-based interrogation of fMRI data sets. Neuroimage 19 (3), 1233-1239.

Maratos, E.J., Rugg, M.D., 2001. Electrophysiological correlates of the retrieval of emotional and non-emotional context. J. Cogn. Neurosci. 13 (7), 877-891. http: //dx.doi.org/10.1162/089892901753165809.
Mather, M., Sutherland, M.R., 2011. Arousal-biased competition in perception and memory. Perspect. Psychol. Sci. 6 (2), 114-133. http://dx.doi.org/10.1177/ 1745691611400234

McGaugh, J.L., 2004. The amygdala modulates the consolidation of memories of emotionally arousing experiences. Annu. Rev. Neurosci. 27 (1), 1-28. http://dx. doi.org/10.1146/annurev.neuro.27.070203.144157.

McGaugh, J.L., 2006. Make mild moments memorable: add a little arousal. Trends Cogn. Sci. 10 (8), 345-347. http://dx.doi.org/10.1016/j.tics.2006.06.001.

McGaugh, J.L., 2013. Making lasting memories: remembering the significant. Proc. Natl. Acad. Sci. 110(Suppl. 2): S10402-S10407 〈http://dx.doi.org/10.1073/pnas. 1301209110).

Moscovitch, M., Rosenbaum, R.S., Gilboa, A., Addis, D.R., Westmacott, R., Grady, C., McAndrews, M.P., Levine, B., Black, S., Winocur, G., Nadel, L., 2005. Functional neuroanatomy of remote episodic, semantic and spatial memory: a unified account based on multiple trace theory. J. Anatomy 207 (1), 35-66. http://dx. doi.org/10.1111/j.1469-7580.2005.00421.x.

Nadel, L., Moscovitch, M., 1997. Memory consolidation, retrograde amnesia and the hippocampal complex. Curr. Opin. Neurobiol. 7 (2), 217-227.

Neisser, U., Harsch, N., 1992. Phantom Flashbulbs: False Recollections of Hearing the News about Challenger. Affect and Accuracy in Recall: Studies of "Flashbulb" Memories. E. W. U. Neisser. Cambridge University Press, New York, NY, US, pp. $9-31$.

Penny, W.D., Holmes, A.P., Friston, K.J., 2003. Random effects analysis. Human Brain Function. Frackowiak, R.S.J., Friston, K.J.C. Frith et al., Academic Press

Phelps, E.A., 2006. Emotion and cognition: insights from studies of the human amygdala. Annu. Rev. Psychol. 57 (1), 27-53. http://dx.doi.org/10.1146/annurev. psych.56.091103.070234.

Richardson, M.P., Strange, B.A., Dolan, R.J., 2004. Encoding of emotional memories depends on amygdala and hippocampus and their interactions. Nat. Neurosci. 7 (3), 278-285. http://dx.doi.org/10.1038/nn1190.

Ritchey, M., Dolcos, F., Cabeza, R., 2008. Role of amygdala connectivity in the persistence of emotional memories over time: an event-related FMRI investigation. Cereb. Cortex 18 (11), 2494-2504. http://dx.doi.org/10.1093/cercor/bhm262.

Schwabe, L., Nader, K., Pruessner, J.C., 2013. $\beta$-Adrenergic blockade during reactivation reduces the subjective feeling of remembering associated with emotional episodic memories. Biol. Psychol. 92 (2), 227-232. http://dx.doi.org/ 10.1016/j.biopsycho.2012.10.003.

Shafer, A.T., Dolcos, F., 2014. Dissociating retrieval success from incidental encoding activity during emotional memory retrieval, in the medial temporal lobe. Front. Behav. Neurosci., 8. http://dx.doi.org/10.3389/fnbeh.2014.00177.

Sharot, T., Delgado, M.R., Phelps, E.A., 2004. How emotion enhances the feeling of remembering. Nat. Neurosci. 7 (12), 1376-1380. http://dx.doi.org/10.1038/ nn1353.

Sharot, T., Verfaellie, M., Yonelinas, A.P., 2007. How emotion strengthens the recollective experience: a time-dependent hippocampal process. PLoS One 2 (10), e1068. http://dx.doi.org/10.1371/journal.pone.0001068.

Smith, A.P., Henson, R.N., Rugg, M.D., Dolan, R.J., 2005. Modulation of retrieval processing reflects accuracy of emotional source memory. Learn. Mem. 12 (5), 472-479. http://dx.doi.org/10.1101/lm.84305.

Smith, A.P., Stephan, K.E., Rugg, M.D., Dolan, R.J., 2006. Task and content modulate amygdala-hippocampal connectivity in emotional retrieval. Neuron 49 (4), 631-638. http://dx.doi.org/10.1016/j.neuron.2005.12.025.

Smith, A.P.R., Henson, R.N.A., Dolan, R.J., Rugg, M.D., 2004. fMRI correlates of the episodic retrieval of emotional contexts. Neuroimage 22 (2), 868-878. http //dx.doi.org/10.1016/j.neuroimage.2004.01.049.

Squire, L.R., 1992. Memory and the hippocampus: a synthesis from findings with rats, monkeys, and humans. Psychol. Rev. 99 (2), 195-231.

Sterpenich, V., Albouy, G., Darsaud, A., Schmidt, C., Vandewalle, G., Dang Vu, T.T., Desseilles, M., Phillips, C., Degueldre, C., Balteau, E., Collette, F., Luxen, A., Maquet P., 2009. Sleep promotes the neural reorganization of remote emotional memory. J. Neurosci. 29 (16), 5143-5152. http://dx.doi.org/10.1523/jneurosci.0561-09.2009.

Strange, B.A., Hurlemann, R., Dolan, R.J., 2003. An emotion-induced retrograde amnesia in humans is amygdala- and $\beta$-adrenergic-dependent. Proc Natl. Acad. Sci. USA 100 (23), 13626-13631. http://dx.doi.org/10.1073/pnas.1635116100.

Strange, B.A., Witter, M.P., Lein, E.S., Moser, E.I., 2014. Functional organization of the hippocampal longitudinal axis. Nat. Rev. Neurosci. 15 (10), 655-669. http://dx. doi.org/10.1038/nrn3785 〈http://www.nature.com/nrn/journal/v15/n10/abs/ nrn3785.html\#supplementary-information>.

Talarico, J., LaBar, K., Rubin, D., 2004. Emotional intensity predicts autobiographical memory experience. Mem. Cogn. 32 (7), 1118-1132. http://dx.doi.org/10.3758/ bf03196886.

Talarico, J.M., Rubin, D.C., 2003. Confidence, not consistency, characterizes flashbulb memories. Psychol. Sci. 14 (5), 455-461. http://dx.doi.org/10.1111/1467-9280.02453.

Takashima, A., Nieuwenhuis, I.L., Jensen, O., Talamini, L.M., Rijpkema, M., Fernández, G., 2009. Shift from hippocampal to neocortical centered retrieval network with consolidation. J Neuroscience 29 (32), 10087-10093.

Todd, R.M., Schmitz, T.W., Susskind, J., Anderson, A.K., 2013. Shared neural substrates of emotionally enhanced perceptual and mnemonic vividness. Front. Behav. Neurosci., 7. http://dx.doi.org/10.3389/fnbeh.2013.00040.

Toffoletto, S., Lanzenberger, R., Gingnell, M., Sundström-Poromaa, I., Comasco, E., 2014. Emotional and cognitive functional imaging of estrogen and progesterone effects in the female human brain: a systematic review. Psychoneuroendocrinology 50, 28-52. http://dx.doi.org/10.1016/j.psyneuen.2014.07.025. Tzourio-Mazoyer, N., Landeau, B., Papathanassiou, D., Crivello, F., Etard, O., Delcroix, 
N., Mazoyer, B., Joliot, M., 2002. Automated anatomical labeling of activations in SPM using a macroscopic anatomical parcellation of the MNI MRI single-subject brain. Neuroimage 15 (1), 273-289. http://dx.doi.org/10.1006/nimg.2001.0978.

Weis, S., Specht, K., Klaver, P., Tendolkar, I., Willmes, K., Ruhlmann, J., Elger, C.E., Fernandez, G., 2004. Process dissociation between contextual retrieval and item recognition. Neuroreport 15 (18), 2729-2733.

Yonelinas, A.P., Ritchey, M., 2015. The slow forgetting of emotional episodic memories: an emotional binding account. Trends Cogn. Sci. 19 (5), 259-267. http://dx.doi.org/10.1016/j.tics.2015.02.009.

Yonelinas, A.P., Dobbins, I., Szymanski, M.D., Dhaliwal, H.S., King, L., 1996. Signaldetection, threshold, and dual-process models of recognition memory: ROCs and Conscious Recollection. Conscious. Cogn. 5 (4), 418-441. http://dx.doi.org/ 10.1006/ccog.1996.0026. 